\title{
A pan-European ring trial to validate an International Standard for detection of Vibrio cholerae, Vibrio parahaemolyticus and Vibrio vulnificus in seafoods
}

Hartnell R.E. ${ }^{1}$, Stockley L. ${ }^{1}$, Keay W. ${ }^{1}$, Rosseau J.-P. ${ }^{2}$, Hervio-Heath Dominique ${ }^{3}$, Van Der Berg H. ${ }^{4}$, Leoni F. ${ }^{5}$, Henigman $U_{.}^{6}$, Denayer S. ${ }^{7}$, Serbruyns B. ${ }^{8}$, Georgsson $F_{.}{ }^{9}$, Krumova G. ${ }^{10}$, Blanco C. ${ }^{11}$, Copin S. ${ }^{12}$, Strauch E. ${ }^{13}$, Wieczorek K. ${ }^{14}$, Britova A. ${ }^{15}$, Hardouin G. ${ }^{16}$, Lombard B. ${ }^{17}$, In'T Veld P. ${ }^{18}$, Leclercq A. ${ }^{19}$, Baker-Austin C. ${ }^{1,}$

${ }^{1}$ European Union Reference Laboratory for Monitoring Bacteriological and Viral Contamination of Bivalve Molluscs, Centre for Fisheries, Environment and Aquaculture Science, Barrack Road, The Nothe, Weymouth DT4 8UB, United Kingdom

${ }^{2}$ Service Commun des Laboratoires Ministère de l' Econopmie et des Finances, Montepellier, France

${ }^{3}$ LNR Microbiologie des Coquillages, Centre Ifremer de Brest, Laboratoire Microbiologie (EMP/MIC), BP 70, 29280 Plouzane, France

${ }^{4}$ National Institute of Public Health and the Environment (RIVM), PO Box 1, Antonie van Leeuwenhoeklaan 9, 3720 BA Bilthoven, The Netherlands

5 Instituto Superiore di Sanita, Ancona, Italy

${ }^{6}$ Institute for food hygiene and bromatology, Veterinary faculty, Gerbiceva 60, SI - 1000, Ljubljana, Slovenia

${ }^{7}$ Scientific Institute of Public Health, Juliette Wytsmanstraat 14, 1050 Brussels, Belgium

${ }^{8}$ Chemiphar n.v, Lieven Bauwensstraat 4, 8200 Brugge, Belgium

9 Icelandic Food and Biotech R\&D, Matvaelarannsoknir Islands, Skulagotu 4, 101 Reykjavik, Iceland

${ }_{11}^{10}$ Food Safety Laboratory, 15 Pencho Slaveikov blvd, Sofia 1606, Bulgaria

${ }^{11}$ Agencia Española de Sguridad Alimentaria, Centro Nacional de Alimentación, Servicio de

Microbiología Alimentaria Carretera de Majadahonda a Pozuelo, Km, 5,2 Majadahonda, 28220 Madrid, Spain

${ }_{12}$ ANSES, Laboratoire des produits de la pêche de Boulogne-sur-mer, Boulevard du Bassin Napoléon, 62200 Boulogne sur Mer, France

${ }^{13}$ Bundesinstitut für Risikobewertung (BfR) Federal Institute for Risk Assessment, Diedersdorfer Weg, 112277 Berlin, Germany

${ }^{14}$ National Veterinary Research Institute, Dept of Food \& Environmental Virology, Partyzantow 57, $24-$ 100 Pulawy, Poland

${ }^{15}$ State Veterinary and Food Institute, Janoskova 1611/58, 02601 Dolny Kubin, Slovakia

${ }_{17}^{16}$ Association Française de Normalisation, Saint-Denis, France

${ }^{17}$ Agence Nationale de Sécurité Sanitaire de l'Alimentation, de l'Environnement et du Travail, MaisonsAlfort, France

${ }^{18}$ Netherlands Food and Consumer Product Safety Authority, Utrecht, The Netherlands

${ }^{19}$ Institut Pasteur, Paris, France 


\begin{abstract}
:
Globally, vibrios represent an important and well-established group of bacterial foodborne pathogens. The European Commission (EC) mandated the Comite de European Normalisation (CEN) to undertake work to provide validation data for 15 methods in microbiology to support EC legislation. As part of this mandated work programme, merging of ISO/TS 21872-1:2007, which specifies a horizontal method for the detection of $V$. parahaemolyticus and $V$. cholerae, and ISO/TS 21872-2:2007, a similar horizontal method for the detection of potentially pathogenic vibrios other than $V$. cholerae and $V$. parahaemolyticus) was proposed. Both parts of ISO/TS 21872 utilized classical culture-based isolation techniques coupled with biochemical confirmation steps. The work also considered simplification of the biochemical confirmation steps. In addition, because of advances in molecular based methods for identification of human pathogenic Vibrio spp. classical and real time PCR options were also included within the scope of the validation. These considerations formed the basis of a multi-laboratory validation study with the aim of improving the precision of this ISO technical specification and providing a single ISO standard method to enable detection of these important foodborne Vibrio spp.. To achieve this aim, an international validation study involving 13 laboratories from 9 countries in Europe was conducted in 2013. The results of this validation have enabled integration of the two existing technical specifications targeting the detection of the major foodborne Vibrio spp., simplification of the suite of recommended biochemical identification tests and the introduction of molecular procedures that provide both species level identification and discrimination of putatively pathogenic strains of $V$. parahaemolyticus by the determination of the presence of theromostable direct and direct related haemolysins. The method performance characteristics generated in this have been included in revised international standard, ISO 21872:2017, published in July 2017.
\end{abstract}

Keywords : Vibrios, Seafood, Prawns, Oysters, Biochemical methods, Real-time PCR, Conventional PCR 


\section{Introduction}

Vibrios are Gram-negative rod-shaped bacteria that are natural constituents of estuarine and marine environments (1). The genus Vibrio contains over 100 described species, and around a dozen of these have been demonstrated to cause infections in humans (2). Typically, Vibrio infections are initiated from exposure to seawater or consumption of raw or undercooked seafood produce $(3,4)$. The species most commonly associated with foodborne infections include V. parahaemolyticus, V. vulnificus and V. cholerae. Several factors underline the importance of these foodborne pathogens from an international perspective. Around $50-85,000$ foodborne Vibrio infections are believed to occur each year in the USA, based on extrapolation data from active and passive epidemiological surveillance systems (5). Compared to other major foodborne pathogens, the number of Vibrio infections also appears to be steadily increasing (6, 7). Recent data from the Centers for Disease Control and Prevention (CDC) indicate that foodborne infections associated with these bacteria has increased significantly in the USA (7). Notably, of all the major bacterial foodborne pathogens (e.g. Salmonella, Listeria, Escherichia coli $\mathrm{O} 157$ and Camplyobacter), vibrios are the only group that are currently increasing in incidence in the USA. Seafood-associated outbreaks are now being reported in geographical areas where these bacteria where once considered absent $(1,6,8,9)$. There is a growing body of evidence to suggest that climate change, coupled to epidemiological and demographic factors are increasing the geographical spread as well as incidence of these pathogens from foodborne sources $(1,6,10)$.

Currently there are no European Union (EU) regulatory microbiological criteria for Vibrio spp. in seafood products traded between Member States or for third country imports. In 2010 Codex published guidelines on the application of general principles for vibrios and seafood produce 
(CAC/GL 73-2010). However, Codex did not provide definitive microbiological criteria, but specified the need for improvements for microbiological approaches in this area. The EU Regulation (EC) No. 2073/2005 sets out the microbiological criteria for foodstuffs produced and traded in Europe, but there are no specific microbiological criteria for Vibrio spp. in this regulation. Although some unilateral risk based controls for imported seafood have been adopted, in principle these are carried out in an ad hoc manner (11). In part the lack of introduction of EU standards for Vibrio spp. in seafoods has been due to the lack of suitable, validated discriminatory methods for Vibrio spp. of major foodborne significance (e.g. $V$. cholerae, V. parahaemolyticus and V. vulnificus (5)), and specifically for toxigenic strains of $V$. parahaemolyticus. The European Commission (EC) mandated the Comite de European Normalisation (CEN) to undertake work to provide validation data for 15 methods in microbiology to support EC legislation. Among these were the 2 existing EN ISO Technical Specifications for the detection of Vibrio spp. of potential public health significance - ISO TS 21872 parts 1 and 2. ISO TS 21872 - 1 provided a detection method for $V$. cholerae and $V$. parahaemolyticus, whereas ISO TS 21872-2 entitled 'Detection of potentially pathogenic Vibrio spp. other than $V$. cholerae and $V$. parahaemolyticus essentially set out a method for detection of $V$. vulnificus. Both ISO TS 21872 parts 1 and 2 were classical culture based qualitative microbiological methods with a suite of biochemical tests for isolate confirmation.

Following preliminary practical evaluation at the European Union Reference Laboratory (EURL), a consultation with AFNOR sub group on Vibrio methods and discussion with experts amongst EU National Reference Laboratory (NRLs) was initiated. A number of improvements to ISO TS 21872 parts 1 and 2 were proposed to relevant sub committees at International Standards Organisation (ISO) and CEN (ISO SC9 and CEN WG6) and subsequently agreed by DG Sante and DG Entr. These comprised merging the two parts to a single standard focusing on major foodborne species V. cholerae, V. parahaemolyticus and V. vulnificus, a revision to include provision for use of PCR based identification of the three species, thermostable direct haemolysin $(t d h)$ and direct related haemolysin $(t r h)$ genes that allow the differentiation of 
pathogenic strains of $V$. parahaemolyticus and minor amendments to biochemical confirmatory tests. A revised standard ISO 21872 was accepted as a New Work Item Proposal (NWIP) by CEN WG6 and this was the within the scope of the validation. This study aimed to validate the consolidated aspects of ISO 21872, utilising collaborative trials of two different seafood matrices (raw bivalve molluscan shellfish and frozen prawns) in order to replace the technical specification with a full, validated EN/ISO standard.

\section{Materials and methods}

\section{Selection of target regions for PCR based species and strain confirmation}

A preliminary collaborative trial amongst 23 NRLs for monitoring viral and bacteriological contamination of bivalve molluscs and other specialist laboratories across the EU was conducted to assist with selection of molecular targets for $V$. parahaemolyticus, $V$. cholerae and V. vulnificus. This included selection and verification of species markers and molecular targets to enable the detection of putative pathogenic strains of $V$. parahaemolyticus possessing $t d h$ and trh genes. Samples were distributed as swabs; reference strains of target and non-target Vibrio spp. were included in the study. A method protocol including well established primer sets and specific running conditions were produced to accompany test samples. Test samples were anonymised and distributed as semi-solid marine agar swabs inoculated with reference strains of target and non-target Vibrio spp.. Swabs were inoculated into $225 \pm 5 \mathrm{ml} \mathrm{ASPW}$ and subject to primary enrichment at $41.5 \pm 1{ }^{\circ} \mathrm{C}$ and $37 \pm 1{ }^{\circ} \mathrm{C}$ for $6 \pm 1 \mathrm{~h}$. One millilitre aliquots of each enrichment were subject to secondary enrichment in $10 \pm 0.5 \mathrm{ml}$ fresh ASPW at $41.5 \pm 1{ }^{\circ} \mathrm{C}$ and $37 \pm 1{ }^{\circ} \mathrm{C}$ for $18 \pm 3 \mathrm{~h}$. Following primary and secondary enrichment, $1 \mu \mathrm{l}$ of each enrichment broth was streaked onto the surface of TCBS and a second plating medium. TCBS plates were incubated at $37 \pm 1{ }^{\circ} \mathrm{C}$ for $24 \pm 3 \mathrm{~h}$; second plating media were incubated according to the manufacturers' instructions. A minimum of two colonies showing typical phenotypic characteristics of Vibrio spp. were sub-cultured onto SNA and incubated at $37 \pm 1{ }^{\circ} \mathrm{C}$ for $24 \pm 3$ 
h. Subsequent cultures were checked visually for purity and subject to oxidase tests and microscopic examination (motility and Gram stain). Oxidase positive, Gram negative, motile isolates were subject to tests for glucose utilisation, lactose and sucrose fermentation, lysine decarboxylation, $\beta$-galactosidase activity, presence of arginine dihydrolase, indole production, ornithine decarboxylase production and growth in $0,2,6,8$, and $10 \% \mathrm{NaCl}$. In parallel, DNA was extracted from a single colony suspended in $500 \mu \mathrm{l}$ of nuclease free water, the bacterial suspension was heated at $95 \pm 1{ }^{\circ} \mathrm{C}$ for $5 \pm 1$ mins and centrifuged at $10,000 \mathrm{x} g$. The resultant supernatant was stored at $<-15{ }^{\circ} \mathrm{C}$ for PCR analysis. Aliquots of $2.5 \mu \mathrm{l}$ of extracted DNA were added to a mastermix containing $10 \mu 1$ reaction buffer, $5 \mu 1 \mathrm{MgCl}_{2}, 0.625 \mu \mathrm{dNTPs}(20 \mathrm{mM}), 0.5$ $\mu \mathrm{l}$ primer (forward and reverse), $0.25 \mu \mathrm{l}$ Taq polymerase and $30.625 \mu 1$ nuclease free water. All samples were subjected to PCR according to the cycling parameters described in for ToxR (12), tdh and trh (13), prVC (14) and $V V H$ (15). Products were visualised on $2 \%$ agarose gels following electrophoresis at 130 volts for 25 - 30 mins.

\section{Selection of laboratories for ring trials}

Recruitment of collaborating laboratories was by open competition. An open call for participants' was launched via the Cefas and EURL websites giving a description of the scope of the proposed work and inviting expressions of interest. Laboratory selection was made by an EURL expert panel and was based upon demonstrable competencies according to the following criteria: 1) demonstrable experience in working with Vibrio spp. and bacteriological testing of seafoods; 2) participation in formal external quality assurance schemes for Vibrio spp. and 3) familiarity with quality assurance procedures and method standardisation.

\section{Collaborative trial 1 - Matrix raw bivalve molluscan shellfish}

\section{Generation of samples, distribution and quality control}


Eight hundred Pacific oysters (Crassostrea gigas) were obtained from a commercial fishery in the UK, and the animals were depurated (purified by holding in 'clean' seawater for 48 hours) at the organising laboratory. Following depuration oysters were removed from tanks and shucked (opened) aseptically. The flesh and intravalvular fluid was homogenised, pooled and split into three large sterile mixer bowls to produce master homogenates. Samples were then spiked with log phase bacterial cultures as follows: 1) V. vulnificus WDCM 00139 low level 2 - 2 x10 $\mathrm{CFU}$ $125 \mathrm{~g}, 2) \mathrm{V}$. parahaemoltycus EURL V05/14 high level $2 \times 10^{6} \mathrm{CFU} / 25 \mathrm{~g}$, and 3), $V$. parahaemoltycus EURL V05/14 low level 2 - 2 x10 CFU /25 g. Master homogenates were mixed thoroughly using a food processor and approximately $35 \mathrm{ml}$ volumes were aliquoted into $50 \mathrm{ml}$ Falcon tubes. Samples were packed in 10L biotherm units with cool packs and distributed by courier under refrigeration conditions according to UN 6673 packing instructions. Samples comprised 8 replicate samples uncontaminated homogenates, 8 samples with inoculated low $V$. vulnificus and high $V$. parahaemolyticus, and 8 samples inoculated with low levels of $V$. parahaemolyticus. The distribution took place on $18^{\text {th }}$ July 2013 thirteen laboratories were sent blind samples labelled 1 - 24. All samples arrived at their destination within 48 hours of dispatch. Separately all participating laboratories received primers, probes and positive and negative control material, as bacterial DNA on dry ice.

\section{Collaborative trial 2 - Matrix cooked, frozen prawns}

\section{Generation of samples, distribution and quality control}

Five kg of cooked, frozen prawns (Penaeus spp.) were purchased from a commercial retail establishment. Samples were defrosted, homogenised (1:2 with $0.1 \%$ peptone) and split into three large sterile mixer bowls to produce master homogenates. Master homogenates were then split into $35 \mathrm{ml}$ volumes in sterile Falcon tubes and frozen at $<-15{ }^{\circ} \mathrm{C}$. Each laboratory received 24 samples packed in $15 \mathrm{~L}$ biotherm units with freezer packs and distributed by courier according to UN 6673 packing instructions. The distribution took place on $18^{\text {th }}$ November 2013 , 
twelve laboratories. Freeze dried cultures were dispatched separately to laboratories with instructions on reconstitution of freeze dried cultures and inoculation of samples prior to analysis. All samples arrived at their destination within 48 hours of dispatch. Separately all participating laboratories received primers, probes and positive and negative control material, as bacterial DNA on dry ice. Target levels for inocula were: 1) V. vulnificus $\approx 1 \times 10^{1} \mathrm{CFU} / 10 \mathrm{~g}$ low level, high level $\left.1 \times 10^{6} \mathrm{CFU} / 10 \mathrm{~g}, 2\right) \mathrm{V}$. parahaemoltycus $1 \times 10^{3} \mathrm{CFU} / 10 \mathrm{~g}$ (low/medium level) and 3) $V$. cholerae $1 \times 10^{3} \mathrm{CFU} / 10 \mathrm{~g}$ low level, high level $1 \times 10^{5} \mathrm{CFU} / 10 \mathrm{~g}$ (low/medium level).

\section{Testing procedures used at diffe rent participating laboratories and analysis of data}

Laboratories involved in the validation exercise were provided with a standard operating procedure (SOP) outlining the steps required for the isolation, identification and verification of strains obtained in distributions 1 (raw bivalve shellfish) and 2 (prawns). In brief, the SOP provided information on the preparation and first enrichment of samples, secondary enrichments, isolation and confirmation of pure cultures, biochemical confirmation and verification using conventional and real-time PCR assays. The SOP also provided a template for the expression of results. The SOP was based on draft iterations of ISO TS 21872, with minor modifications (Figure 1). The PCR and real-time PCR methods described in the SOP and used for confirmatory purposes were based on a range of well-established published assays for $V$. cholerae (14), V. parahaemolyticus $(12,13)$ (including putative pathogenicity maker genes $t d h$ and $t r h)(13,18)$ and $V$. vulnificus $(15,16$ (with minor modification)) thus meeting the criterion established in the draft standard that primer (and hydrolysis probe) sequences shall be published in peer-review journals and verified against a broad range of target and non-target strains running conditions. PCR set up and primer and probe sequences were subsequently based on these published studies. Data returned to the EURL for analysis was subsequently collated and analysed per distribution, with performance characteristics gathered. Sensitivity and specificity 
were calculated according to the following criteria. The sensitivity is defined as the number of samples found positive divided by the number of samples tested at a given level of contamination. The results are thus dependent on the level of contamination of the sample. The specificity is defined as the number of blank samples found negative divided by the number of blank samples tested found negative. Criteria for determining both specificity and sensitivity were defined by ISO statistics working group and are shared amongst a number of other published ISO documents in mandate M381. Currently estimated as per g, $95 \%$ confidence intervals in parentheses. Performance characteristics such as limit of detection (LOD) and $\mathrm{LOD}_{50}$ of submitted data from participating laboratories was analysed using a Probability of Detection (POD) approach essentially as previously described (17). The values of the performance characteristics derived from this interlaboratory study are shown per bacterial determinant and type of sample in Tables 1 - 3. Identification of target Vibrio spp. were by biochemical, conventional and/or real real-time PCR. Real-time PCR for identification of $V$. cholerae was tested in this international interlaboratory study but data generated were not reliable and subsequently omitted (data not shown).

\section{Results}

\section{Selection of laboratories for ring trials}

Twenty-three laboratories were recruited into the initial EURL trials to select molecular target regions for identification of $V$. parahaemolyticus, $t d h$, trh genes, $V$. vulnificus and $V$. cholerae. Of these, 15 responded to the open call for participation in the validation exercise. Thirteen laboratories were selected from assessment of the expressions of interest provided detailing their relevant experience against the selection criteria. Two laboratories were not considered to have demonstrated sufficient experience in the application of molecular methods for the detection of foodborne bacterial pathogens or did not have demonstrable experience of relevant quality 
assurance or standardisation. Consequently 13 laboratories were selected for inclusion in the validation.

\section{Collaborative trial 1 - Matrix raw bivalve molluscan shellfish}

A large dataset was generated from the interlaboratory trial exercises carried out as part of the CEN validation study. These data were used to determine the performance characteristics of the method in matrix samples. In the evaluation of these data, biochemical and molecular isolate identification testing approaches were considered together. In a small number of cases isolate identification using biochemical and molecular approaches did not yield corresponding results (data not shown). In these cases, samples were considered positive if the laboratory recorded that either one of the colony identification approaches employed following primary and secondary enrichment at either $37^{\circ} \mathrm{C}$ or $41.5^{\circ} \mathrm{C}$ yielded a positive result. This approach was also employed for assessment of Collaborative trial 2. Thirteen participating laboratories took part in the raw bivalve mollusc distribution (Tables $1-3$ ). This number was reduced to 10 participating laboratories following the evaluation of data. Entire datasets were excluded from 3 laboratories, as complete sets of results for both biochemical and molecular identification procedures for isolated colonies were not provided. Eighty samples of low-level spiked material and high-level spiked material (V. parahaemolyticus) and eighty blank samples were used for data appraisal purposes (Table 1). The highest $\%$ sensitivity results obtained were in the highlevel contamination sample $\left(2 \times 10^{6} \mathrm{CFU} / 25 \mathrm{~g}\right.$ V. parahaemolyticus $)$ where $100 \%$ of samples gave positive results. Lower level contamination samples $\left(\begin{array}{llllll}2 & \text { x10 } & \text { CFU/25 } & \mathrm{g} & V \text {. }\end{array}\right.$ parahaemolyticus) generated positive results in almost three quarters of tested samples (72.5 $\%)$. with blank samples indicating sensitivity levels of $90 \%$. Specificity was calculated at 111 $\%$ across all samples, exceedance of $100 \%$ for specificity resulted from a low number of positive identifications in 'blank' samples (Table 1). The $\mathrm{LOD}_{50}(\mathrm{CFU} / \mathrm{g})$ was determined to be 0.43 , with a range of $0.32-0.57$. Lower overall levels of sensitivity were observed using testing 
methods for $V$. vulnificus, which generated positive results in only $37.5 \%$ of samples from the low-level contamination scenario $(2 \mathrm{x} 10 \mathrm{CFU} / 25 \mathrm{~g}$ V. parahaemolyticus, Table 1$)$. This observation was corroborated following calculation of the $\mathrm{LOD}_{50}$ which showed a notably higher obtained value (12.31 CFU/g - range 8.46 - 17.91) compared to similar inocula levels for V. parahaemolyticus. Irrespective, good species-specificity was obtained, with $77.5 \%$ of samples providing expected results. An additional aspect of the validation exercise was to obtain relevant performance characteristics of tests to determine virulence markers used for $V$. parahaemolyticus, using published assays for both conventional and real-time PCR assays for $t d h$ and $t r h$ ). A smaller subset of participating laboratories provided results for detection of $t d h$ and $t r h$ genes in strains isolated from the main validation samples (9 laboratories provided data on real-time PCR and 7 for conventional PCR), the data indicate that both methods perform well in terms of discriminating potentially pathogenic strains based upon the presence of either or both thermostable haemolysin genes (Table 2). Similar levels of \% specificity and sensitivity were observed for conventional PCR methods for $t d h$ and $t r h$ (around $90 \%$ ), although slightly lower sensitivity was observed using real-time PCR for $t d h$ (Table 2). The real-time $t r h$ assay used during this validation exercise did not perform reliably and was subsequently omitted from the final dataset.

\section{Collaborative trial 2 - Matrix cooked, frozen prawns}

Eleven of the original thirteen participating laboratories undertook a second distribution for detection of V. cholerae, $V$. vulnificus and $V$. parahaemolyticus in cooked frozen prawns. Of the eleven datasets returned, data from one laboratory were excluded from the assessment as substantive deviations from protocols for real time identification were reported. Analysis of the remaining dataset revealed good sensitivity (92.5\% in both low and high level spiked samples), and good specificity $(84 \%)$ for $V$. cholerae with $\mathrm{LOD}_{50}$ results $(11.34 \mathrm{CFU} / 25 \mathrm{~g}$ - range 6.26 to 20.56) (very similar to the reported $\mathrm{LOD}_{50}$ for $V$. vulnificus in raw oysters. No V. cholerae 
strains were reported from blank samples of prawns, giving sensitivity $\%$ of $100 \%$ (Table 3). Less satisfactory performance was obtained in prawn samples spiked with either low $\left(1 \times 10^{2}\right.$ CFU/25 g) or moderate- high $\left(1 \times 10^{4} \mathrm{CFU} / 25 \mathrm{~g}\right)$ levels of $V$. vulnificus (Table 3$)$ where assessment indicated sensitivity of between 61.25 and $71.5 \%$ in low and high level contaminated samples respectively and specificity of $60 \%$. A substantively higher LOD $_{50}(81.2$ CFU - range 63.39 - 101.2) was also observed in these samples. These data contrast with the performance characteristics obtained for $V$. parahaemolyticus in cooked, frozen prawns (Table 3), which showed $100 \%$ sensitivity in blank and low-moderate level $\left(1 \times 10^{3} \mathrm{CFU} / 25 \mathrm{~g}\right)$ spiked samples and demonstrated that V. parahaemolyticus could be accurately identified in $99 \%$ of tested samples (Table 3).

\section{Discussion}

Globally, the species $V$. parahaemolyticus, $V$. cholerae and $V$. vulnificus represent the most important bacterial pathogens associated with the consumption of seafood produce $(7,19,20)$. In many countries, a food safety criteria or guidance have been introduced to limit the risks of exposure of consumers of seafood to Vibrio spp. (21-23). Some such interventions are based upon the introduction of testing regimes that determine food safety criteria for Vibrio spp. in certain foodstuffs. In the EU 2001, the former Scientific Committee on Veterinary Measures Related to Public Health (now European Food Safety Authority (EFSA)) published an opinion on $V$. parahaemolyticus and $V$. vulnificus in raw seafood (24). With respect to methods, it was concluded that the sensitivity, specificity and pathogenicity determination of the different methods in use was highly variable and thus the results of studies were difficult to compare. It was recommended inter alia that the elaboration of methods that enabled detection, enumeration and virulence characterisation of $V$. parahaemolyticus and $V$. vulnificus in seafoods should be a priority. Such methods were required in order to enable robust assessment of public health risks associated with Vibrio spp. in seafoods, which may ultimately be used to inform decisions on 
efficacy of control measures or to support future microbiological criteria. This validation exercise therefore was intended, in part, to generate data to enable improvements to existing ISO Technical Specifications for Vibrio spp., which would target the primary Vibrio spp. of human health relevance in seafoods (V. parahaemolyticus and $V$. vulnificus) and enable virulence characterisation of $V$. parahaemolyticus. In addition, because there is clear evidence that $V$. cholerae can be associated with consumption of seafoods $(25,26)$, and its detection was included within ISO TS 21872-1, it was also incorporated into the scope of the validation exercise.

The objective of this work was to combine and simplify ISO TS 21872 Microbiology of the food and animal feeding stuffs - Horizontal method for the detection of potentially enteropathogenic Vibrio spp. Part 1 Detection of V.parahaemolyticus and Vibrio cholerae and Part 2 Detection of species other than V. parahaemolyticus and Vibrio cholerae and, to introduce the ability to use molecular identification approaches following colony isolation from seafood matrices. The introduction of molecular approaches was intended to enable both identification of target Vibrio spp. to the species level, and to facilitate detection of the putative pathogenicity markers $t d h$ and $t r h$ of $V$. parahaemolyitcus. Many authors have reported on the difficulties in the interpretation of biochemical identification methods for Vibrio spp. from environmental sources (including those deriving from seafoods) (27-31) and it has long been recognised that methods for vibrios of human health relevance require improvements and refinements (11), particularly given their emerging nature internationally. The work presented here provides validation data to support the elaboration of a single standardised methodological framework for the detection of V. parahaemolyticus, V. vulnificus and V. cholerae in a range of seafood matrices.

Simplification of recommended number of biochemical identification tests included in the revision enables laboratories to maximise resources and to target the tests which provide the 
most discriminatory abilities. Atypical biochemical test results amongst Vibrio spp. isolated from the environment have been reported by several authors $(27,29)$. For $V$. parahaemolyticus and $V$. vulnificus positive results for production of decarboxylase enzymes (ornithine decarboxylase test) can be variable, and are influenced by the effects of salt concentration in plating media (30). For targeting of $V$. parahaemolyticus, $V$. vulnificus and $V$. cholerae, it was considered that inclusion of lysine decarboxylase and arginine dihydrolase tests provided sufficient discriminatory ability. Similarly, sugar fermentation patterns based on interpretation of the saline triple sugar iron agar test did not generate additional lucidity in the discrimination of V. parahaemolyticus, V. vulnificus and V. cholerae (data not shown), with substantial variability in positivity reported previously for V. vulnificus (26).

The results of validation exercise verified the utility of a number of species-specific testing reliant on PCR (or real time PCR) that had been previously published and that had performed well in earlier EURL ring trials. Although not considered explicitly within the scope of the validation, which considered either biochemical or molecular isolate identification as permissible, the data indicated that for identification of colonies molecular species identification methods for $V$. parahaemolyticus, $V$. vulnificus and $V$. cholerae were less subjective and required less specialist experience to interpret. This finding is in accordance with numerous reports supporting the use of molecular identification for this cryptic species, especially in respect of to environmental isolations $(12,13,15,16,18,26,29,32)$. Whilst molecular identification methods were broadly successful problems were identified with 2 real-time PCR assays selected for inclusion within the validation (real time PCR assays for V. cholerae (33) and trh for $V$. parahaemolyticus (18)), where data demonstrated poor performance methodologies were excluded from the annexes of the published ISO standard as the methods could not be recommended on the basis of the validation. Further work in determining the factors underlining the poor performance is required to elucidate this fully. The validation data demonstrating the utility of $t r h$ and $t d h$ PCR testing methods (Table 2) using methods described previously (13) represents a significant enhancement to the existing technical specification in 
that it enables non-specialist/non-reference laboratories to discriminate between pathogenic and non-pathogenic strains of $V$. parahaemolyticus. Adoption of this approach will enable more rapid assessment of the potential public health risks associated with isolation of $V$. parahaemolyticus in seafoods.

The data generated in this multi-laboratory study enabled the publication of performance characteristics (sensitivity, specificity and $\mathrm{LOD}_{50}$ ). Concordance, i.e. the percentage of all data pairings of duplicates giving the same results used as a measure of reproducibility for qualitative analysis, was calculated according to ISO 16140:2003 (Microbiology of food and animal feeding stuffs -- Protocol for the validation of alternative methods). For the two trials concordance was calculated as $87.7 \%, 62.5 \%$ and $92.5 \%$ for $V$. parahaemolyticus, $V$. vulnificus and V. cholerae respectively. Performance data Vibrio spp. is sparse, however, for $V$. parahaemolyticus and $V$. cholerae $\mathrm{LOD}_{50}$, sensitivity and specificity for seafoods matrices were broadly similar to other bacterial - matrix combinations reported elsewhere (34). For $V$. vulnificus method performance was below that generally expected for bacteriological culture based methods with respect to $\operatorname{LOD}_{50}$. This finding was not surprising and confirms the assertions in a number of studies that report the difficulties in isolation of $V$. vulnificus from environmental samples containing mixed competing microbial flora (reviewed in (35)).

In conclusion, the data generated from this pan-European ring trial to validate an international standard method for the detection of $V$. cholerae, V. parahaemolyticus and V. vulnificus in seafoods represents an important technical advance. This work allows for a more streamlined and simplified standard method that allows for the first time, the simultaneous identification of three Vibrio spp. of major foodborne significance. This advance also improves the accuracy of identification, the simplification of biochemical tests and introduction of molecular species markers for these pathogens. Critically, for the first time, this method facilitates non-specialist and/or non-reference laboratories to discriminate between pathogenic and non-pathogenic strains of $V$. parahaemolyticus. This method does not provide a basis for the quantitative 
evaluation of samples. So, in the case of $V$. parahaemolyticus, or in situations where enumeration of toxigenic strains is deemed important, further methodological work is clearly required. Current work involves refining a quantitative approach, based on real-time PCR coupled to MPN based approach (36), as a possible quantitative testing platform.

\section{Acknowledgements}

The validation of International Standard EN ISO 15216-1 was carried out under the framework of European Mandate No. M381 of DG SANTE and DG GROW (European Commission). Authors wish to thank all participants in the collaborative trials from the following in stitutes: European Union Reference Laboratory for Monitoring Bacteriological and Viral Contamination of Bivalve Molluscs, Centre for Fisheries, Environment and Aquaculture Science, Barrack Road, The Nothe, Weymouth, United Kingdom, DT4 8UB. Service Commun des Laboratoires Ministère de l' Econopmie et des Finances, Montepellier, France. LNR Microbiologie des Coquillages, Centre Ifremer de Brest, Laboratoire Microbiologie (EMP/MIC), BP 70, 29280 Plouzane, France. National Institute of Public Health and the Environment (RIVM), PO Box 1, Antonie van Leeuwenhoeklaan 9, 3720 BA Bilthoven The Netherlands. Instituto Superiore di Sanita, Ancona, Italy. Institute for food hygiene and bromatology, Veterinary faculty, Gerbiceva 60, SI - 1000, Ljubljana, Slovenia. Scientific Institute of Public Health, Juliette Wytsmanstraat 14, 1050 Brussels, Belgium. Chemiphar n.v, Lieven Bauwensstraat 4, 8200 Brugge, Belgium. Icelandic Food and Biotech R\&D, Matvaelarannsoknir Islands, Skulagotu 4, 101 Reykjavik, Iceland. Food Safety Laboratory, 15 Pencho Slaveikov blvd, Sofia 1606, Bulgaria. Agencia Española de Sguridad Alimentaria. Centro Nacional de Alimentación. Servicio de Microbiología Alimentaria Carretera de Majadahonda a Pozuelo. Km, 5,2 Majadahonda. 28220 Madrid. Spain. ANSES, Laboratoire des produits de la pêche de Boulogne-sur-mer, Boulevard du Bassin Napoléon, 62200 Boulogne sur Mer, France. Bundesinstitut für Risikobewertung (BfR) Federal Institute for Risk Assessment, Diedersdorfer 
Weg 112277, Berlin, Germany. National Veterinary Research Institute, Dept of Food \& Environmental Virology Partyzantow 57, 24-100 Pulawy, Poland. State Veterinary and Food Institute, Janoskova 1611/58 02601 Dolny Kubin, Slovakia.

\section{References}

1. Baker-Austin C, Trinanes J, Gonzalez-Escalona N, Martinez-Urtaza J. Non-Cholera Vibrios: The Microbial Barometer of Climate Change. Trends Microbiol [Internet]. 2016 Nov 14; Available from: http://dx.doi.org/10.1016/j.tim.2016.09.008

2. Austin B. Bacteria pathogens of marine fish. In: Oceans and Health: Pathogens in the Marine Environment. 2005. p. 391-413.

3. Altekruse SF, Bishop RD, Baldy LM, Thompson SG, Wilson S a, Ray BJ, et al. Vibrio gastroenteritis in the US Gulf of Mexico region: the role of raw oysters. Epidemiol Infect. 2000;124(3):489-95.

4. Iwamoto M, Ayers T, Mahon BE, Swerdlow DL. Epidemiology of seafood-associated infections in the United States. Clin Microbiol Rev. 2010;23(2):399-411.

5. Scallan E, Hoekstra RM, Angulo FJ, Tauxe R V., Widdowson MA, Roy SL, et al. Foodborne illness acquired in the United States-Major pathogens. Emerg Infect Dis. 2011;17(1):7-15.

6. Martinez-Urtaza J, Bowers JC, Trinanes J, DePaola A. Climate anomalies and the increasing risk of Vibrio parahaemolyticus and Vibrio vulnificus illnesses. Food Res Int

[Internet]. 2010;43(7):1780-90. Available from: http:/dx.doi.org/10.1016/j.foodres.2010.04.001

7. Newton A, Kendall M, Vugia DJ, Henao OL, Mahon BE. Increasing rates of vibriosis in the United States, 1996-2010: Review of surveillance data from 2 systems. Clin Infect Dis. 2012;54(SUPPL.5):391-5.

8. Gonzalez-Escalona N, Cachicas V, Acevedo C, Rioseco ML, Vergara JA, Cabello F, et al. Vibrio parahaemolyticus diarrhea, Chile, 1998 and 2004. Emerg Infect Dis. 2005 Jan;11(1):129-31.

9. Martinez-Urtaza J, Baker-Austin C, Jones JL, Newton AE, Gonzalez-Aviles GD, 
DePaola A. Spread of Pacific Northwest Vibrio parahaemolyticus strain. N Engl J Med [Internet]. 2013;369(16):1573-4. Available from: http//www.ncbi.nlm.nih.gov/pubmed/24131194

10. Vezzulli L, Colwell RR, Pruzzo C. Ocean Warming and Spread of Pathogenic Vibrios in the Aquatic Environment. Microb Ecol. 2013;65(4):817-25.

11. Baker-Austin C, Stockley L, Rangdale R, Martinez-Urtaza J. Environmental occurrence and clinical impact of Vibrio vulnificus and Vibrio parahaemolyticus: A European perspective. Environ Microbiol Rep. 2010;2(1):7-18.

12. Kim YBU, Okuda JUN, Matsumoto C, Takahashi N, Hashimoto S, Nishibuchi M. Note_ Identification of Vibrio parahaemolyticus Strains at the Species Level by PCR Targeted to the toxR Gene. 1999;37(4):1173-7.

13. Bej AK, Patterson DP, Brasher CW, Vickery MCL JD, C K. Detection of total and hemolysin producing Vibrio parahaemolyticus in shellfish using multiplex PCR amplification of tl, tdh, and trh. J Microbiol Methods. 1999;36:215-25.

14. Chun J, Huq A, Colwell RR. Analysis of 16S-23S rRNA intergenic spacer regions of Vibrio cholerae and Vibrio mimicus. Appl Environ Microbiol. 1999;65(5):2202-8.

15. Hill WE, Keasler SP, Trucksess MW, Feng P, Kaysner CA, Lampel KA. Polymerase chain reaction identification of Vibrio vulnificus in artificially contaminated oysters. Appl Environ Microbiol. 1991;57(3):707-11.

16. Campbell MS, Wright AC. Real-Time PCR Analysis of Vibrio vulnificus from Oysters. 2003;69(12):7137-44.

17. Wilrich C, Wilrich P-T. Estimation of the POD function and the LOD of a qualitative microbiological measurement method. J AOAC Int. 2009;92(6):1763-72.

18. Nordstrom JL, Vickery MCL, Blackstone GM, Murray SL, DePaola A. Development of a multiplex real-time PCR assay with an internal amplification control for the detection of total and pathogenic Vibrio parahaemolyticus bacteria in oysters. Appl Environ Microbiol. 2007;73(18):5840-7.

19. Morris JG. Cholera and other types of vibriosis: a story of human pandemics and oysters on the half shell. Clin Infect Dis. 2003;37(2):272-80.

20. Potasman I, Paz A, Odeh M. Infectious outbreaks associated with bivalve shellfish 
consumption: a worldwide perspective. Clin Infect Dis. 2002;35(8):921-8.

21. Toyofuku H. Vibrio parahaemolyticus Risk Management in Japan. In: Sauvé G, editor. Molluscan Shellfish Safety [Internet]. Dordrecht: Springer Netherlands; 2014. p. 129-36. Available from: http://dx.doi.org/10.1007/978-94-007-6588-7_11

22. FDA. National Shellfish Sanitation Program Guide for the Control of Molluscan Shellfish. Natl Shellfish Sanit Progarm. 2015;547.

23. Baker-Austin C, Stockley L, Rangdale R, Martinez-Urtaza J. Environmental occurrence and clinical impact of Vibrio vulnificus and Vibrio parahaemolyticus: A European perspective. Vol. 2, Environmental Microbiology Reports. 2010. p. 7-18.

24. SCVMPH. Opinion of the Scientific Committee on Veterinary Measures Relating to Public Health on Vibrio Vulnificus and Vibrio Parahaemolyticus. 2001.

25. Donovan TJ, van Netten P. Culture media for the isolation and enumeration of pathogenic Vibrio species in foods and environmental samples. Int J Food Microbiol. 1995 Jun;26(1):77-91.

26. K.A BGI and FR. Cowan and Steel's manual for the identification of medical bacteria. Cambridge University Press; 1999.

27. A. AM and B. A set of keys for biochemical identification of environmental Vibrio species. Appl Environ Microbiol. 1994;76:79-85.

28. Janda JM, Powers C, Bryant RG, Abbott SL. Current perspectives on the epidemiology and pathogenesis of clinically significant Vibrio spp. Clin Microbiol Rev. 1988;1(3):245-67.

29. Croci L, Suffredini E, Cozzi L, Toti L, Ottaviani D, Pruzzo C, et al. Comparison of different biochemical and molecular methods for the identification of Vibrio parahaemolyticus. J Appl Microbiol. 2007 Jan;102(1):229-37.

30. Brenner DJKNR and SJT. Bergey's Manual of Systematic Bacteriology. 2nd editio. Springer; 2005.

31. Ottaviani D, Masini L, Bacchiocchi S. A biochemical protocol for the isolation and identification of current species of Vibrio in seafood. J Appl Microbiol. 2003;95(6):1277-84.

32. Chen $\mathrm{CY}, \mathrm{Wu} \mathrm{KM}$, Chang YC, Chang CH, Tsai HC, Liao TL, et al. Comparative 
genome analysis of Vibrio vulnificus, a marine pathogen. Genome Res. 2003 Dec;13(12):2577-87.

33. Lyon WJ. TaqMan PCR for Detection of Vibrio in Pure Cultures, Raw Oysters, and Synthetic Seawater TaqMan PCR for Detection of Vibrio cholerae O1, O139, Non-O1, and Non-O139 in Pure Cultures, Raw Oysters, and Synthetic Seawater $\uparrow$. Society. 2001;67(10):4685-93.

34. Risk M, Series A. Selection and application of methods for the detection and enumeration of human-pathogenic halophilic.

35. Harwood VJ, Gandhi JP, Wright AC. Methods for isolation and confirmation of Vibrio vulnificus from oysters and environmental sources: a review. J Microbiol Methods. 2004 Dec;59(3):301-16.

36. Cantet F, Hervio-Heath D, Caro A, Le Mennec C, Monteil C, Quéméré C, et al. Quantification of Vibrio parahaemolyticus, Vibrio vulnificus and Vibrio cholerae in French Mediterranean coastal lagoons. Res Microbiol. 2013;164(8).

37. ISO C. EN ISO 16140: Microbiology of food and animal feeding stuffs - Protocol for the validation of alternative methods. 2016 . 
Figure 1. Diagram of procedure for the detection of enteropathogenic Vibrio parahaemolyticus, Vibrio cholerae and Vibrio vulnificus

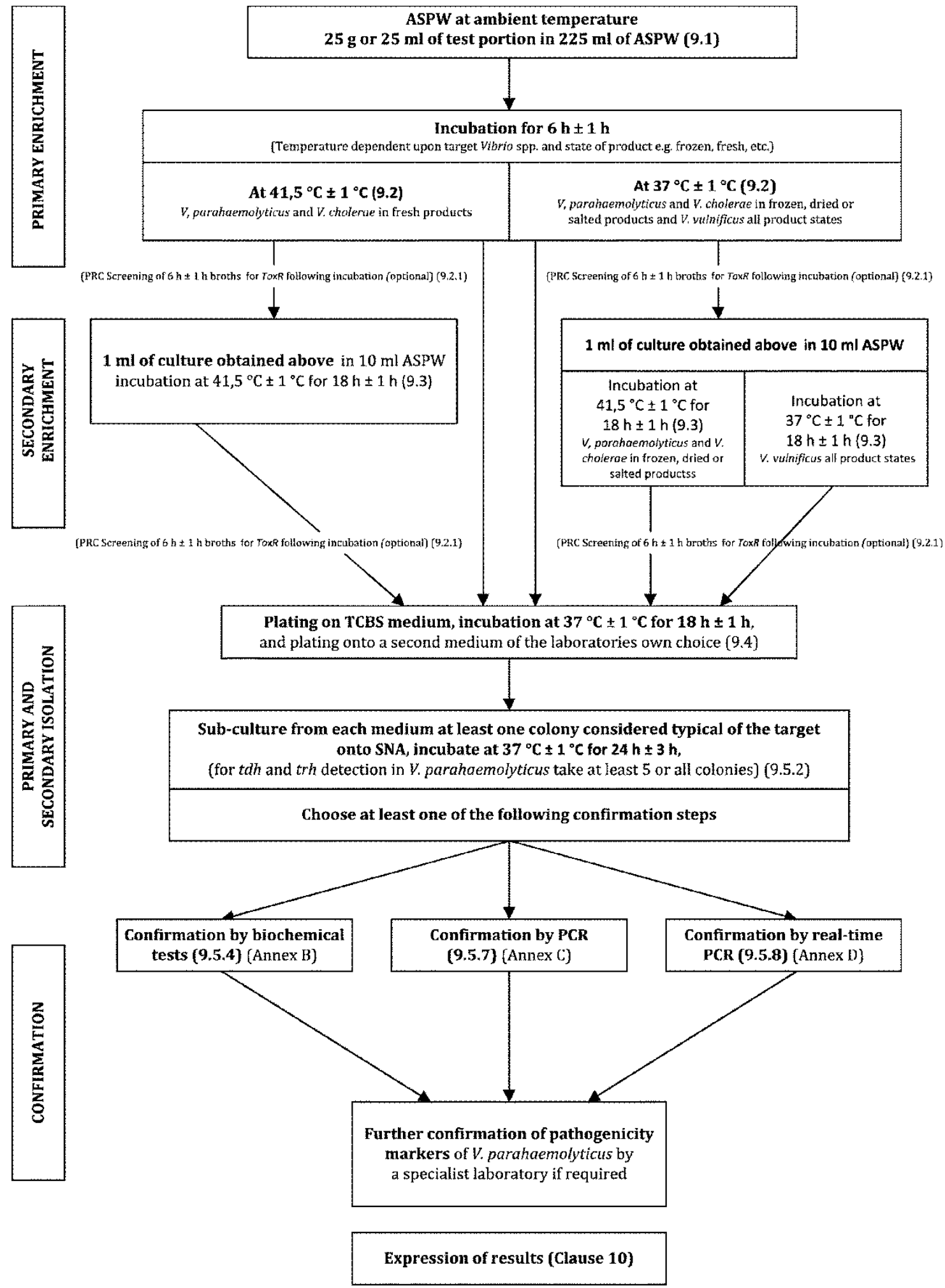


Table 2 - Results of data analysis obtained for tdh and trh for Vibrio parahaemolyticus in raw oysters

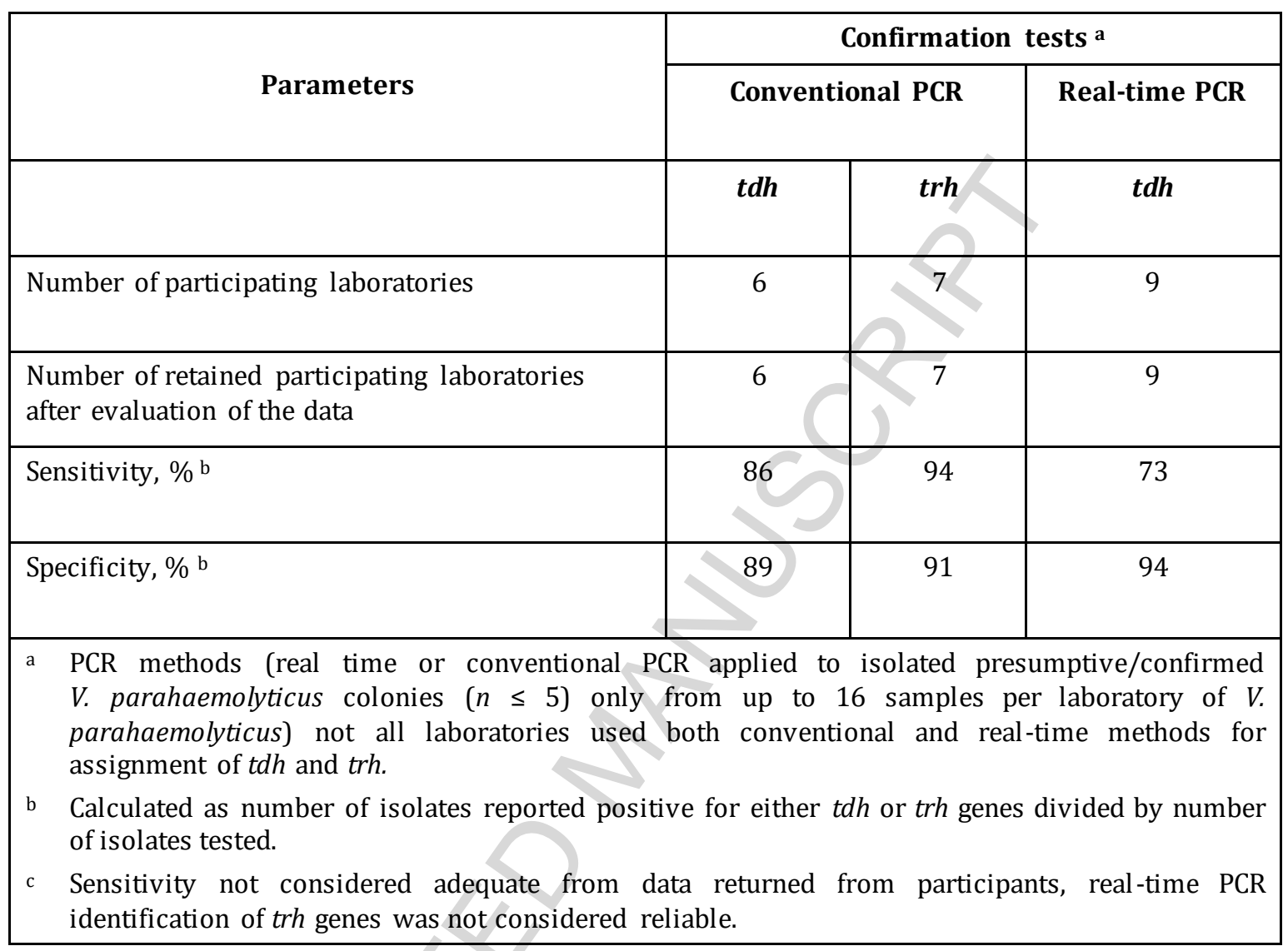


